\title{
Front Resolving Observational Network with Telemetry (FRONT)
}

\author{
James O'Donnell, Dan Codiga, Christopher Edwards \\ Dept. of Marine Sciences \\ University of Connecticut \\ 1084 Shennecossett Road \\ Groton, CT 06340
}

\author{
Phone: (860) 405-9171 Fax: (860) 405-9153 email: james.odonnell@uconn.edu \\ John Marshall, Michael J. Follows \\ Dept. of Earth, Atmospheric and Planetary Sciences \\ Massachusetts Institute of Technology \\ Cambridge, MA 02139 \\ Phone: (617) 253-9615 email: marshall@gulf.mit.edu \\ Scott M. Gallager, Heidi M. Sosik, Cabell S. Davis \\ Woods Hole Oceanographic Institution \\ Woods Hole, MA 02543 \\ Phone: (508) 289-2783 email: sgallager@whoi.edu
}

Award Number: N00014-99-1-1020

http://nopp.uconn.edu

\section{LONG-TERM GOALS}

The Front Resolving Observational Network with Telemetry (FRONT) experiment is designed to evaluate the feasibility of making useful coastal ocean forecasts with a coarse array of instruments that telemeter physical and biological data in near-real time and assimilative numerical models. The FRONT products will be compared to observations obtained during intensive survey campaigns.

\section{OBJECTIVES}

The FRONT site is a portion of continental shelf south of the eastern end of Long Island, New York. Historical observations indicate that variable bathymetry and strong tides interact with energetic windand buoyancy-forced motions to produce recurring fronts. Our objective is to develop and test the observation system in this complex dynamical regime.

Technical objectives include coordination and telemetry of real-time data streams from a combination of in situ instruments including ADCPs, profiling CTDs, and an Autonomous Vertically Profiling Plankton Observatory (AVPPO). Shore-based CODAR will provide real-time measurement of surface current. Satellite data will provide large-scale coverage of sea-surface temperature and color.

The model is based on a high spatial resolution version of the MIT general circulation model (MITgcm). Our objectives are (1) to quantitatively understand the physical dynamics in the study area; (2) identify and illustrate the processes and interactions controlling the broad-scale chlorophyll distributions observed by the in-situ biological profile data and the remote SeaWiFS color sensor, and (3) the influence of frontal scale processes and interactions on biological dynamics and distributions in the region. 


\section{Report Documentation Page}

Form Approved

OMB No. 0704-0188

Public reporting burden for the collection of information is estimated to average 1 hour per response, including the time for reviewing instructions, searching existing data sources, gathering and maintaining the data needed, and completing and reviewing the collection of information. Send comments regarding this burden estimate or any other aspect of this collection of information,

including suggestions for reducing this burden, to Washington Headquarters Services, Directorate for Information Operations and Reports, 1215 Jefferson Davis Highway, Suite 1204, Arlington

VA 22202-4302. Respondents should be aware that notwithstanding any other provision of law, no person shall be subject to a penalty for failing to comply with a collection of information if it

does not display a currently valid OMB control number.

1. REPORT DATE

30 SEP 2001

4. TITLE AND SUBTITLE

Front Resolving Observational Network with Telemetry (FRONT)

6. $\operatorname{AUTHOR}(\mathrm{S})$

7. PERFORMING ORGANIZATION NAME(S) AND ADDRESS(ES)

Dept. of Marine Sciences,„University of Connecticut,„1084 Shennecossett Road,Groton,,CT,06340

9. SPONSORING/MONITORING AGENCY NAME(S) AND ADDRESS(ES)

3. DATES COVERED

00-00-2001 to 00-00-2001

5a. CONTRACT NUMBER

5b. GRANT NUMBER

5c. PROGRAM ELEMENT NUMBER

5d. PROJECT NUMBER

5e. TASK NUMBER

5f. WORK UNIT NUMBER

8. PERFORMING ORGANIZATION

REPORT NUMBER

10. SPONSOR/MONITOR'S ACRONYM(S)

11. SPONSOR/MONITOR'S REPORT

NUMBER(S)

12. DISTRIBUTION/AVAILABILITY STATEMENT

Approved for public release; distribution unlimited

13. SUPPLEMENTARY NOTES

14. ABSTRACT

15. SUBJECT TERMS

16. SECURITY CLASSIFICATION OF:

a. REPORT

unclassified b. ABSTRACT

unclassified c. THIS PAGE

unclassified
17. LIMITATION OF ABSTRACT

Same as

Report (SAR)
18. NUMBER 19a. NAME OF

OF PAGES RESPONSIBLE PERSON

6

Standard Form 298 (Rev. 8-98) Prescribed by ANSI Std Z39-1 


\section{APPROACH}

Data telemetry and instrument control will be accomplished with an acoustic underwater communications network that interfaces with buoy-mounted cellular telephones to connect the subsurface network to the internet. The FRONT network features multiple acoustic modems connected in a topology that can tolerate failure or loss of individual elements. Joe Rice of Space and Naval Warfare Systems Center at San Diego (SSC-SD) is working toward this goal. Rice is a PI on the FRONT team with an independent award. Dan Codiga (University of Connecticut) is coordinating the physical oceanographic instrumentation with the Navy's acoustic network.

Scott Gallager, Heidi M. Sosik, and Cabell S. Davis (all at Woods Hole Oceanographic Institution) will be deploying the AVPPO, integrating it into the acoustic network, and analyzing and preparing the data for integration into the biological assimilative model.

Christopher Edwards and Philip Bogden (prior to his departure to GOMOOS) in collaboration with John Marshall and Michael Follows (Massachusetts Institute of Technology) have set up the MITGCM for the FRONT domain. Mick Follows and Stephanie Dutkiewicz (MIT) in consultation with biologists Gallager, Sosik, and Davis at WHOI, and Petra Stegmann (University of Rhode Island) are embedding their regional ecosystem model in the circulation model. With the combined modeling effort we will synthesize the physical and biological data to advance our understanding of the biological interactions on frontal scales.

Jim O'Donnell (UConn) is coordinating ship surveys with ADCPs and CTDs designed to provide data for verification and testing of the observation system in regions of strong frontal activity. O'Donnell is also developing a new instrument array designed to resolve the small-scale frontal features that will remain under sampled by the moored instruments and unresolved by the models. These frontal scale observations are being conducted in concert with Ed Levine's (Naval Undersea Warfare Center) turbulence measurements obtained from the REMUS ROV. These activities will test the accuracy of the model's sub-grid scale parameterizations.

Remotely sensed surface current maps from CODAR (Dave Ullman, URI) will provide an important supplement to the in situ array. Don Barrick and Belinda Lipa (both with CODAR Ocean Sensors, Ltd.) will assist in the improvement of signal-processing algorithms for using CODAR. This will help CODAR become an integral part of the assimilative models. Ullman and O'Donnell are evaluating the uncertainties in CODAR current estimates by comparing them to ship mounted ADCP survey data and USCG R\&D Center (Jennifer Dick-O'Donnell and Arthur Allen) drifter observations when these become available.

\section{WORK COMPLETED}

During the second year of the project we have completed the acquisition of instruments and the design and fabrication of deployment hardware. This includes two acoustic modem-cellular telephone network gateway buoys; seven trawl-resistant ADCP bottom frames with acoustic modem transceivers; and two moored CTD profilers with acoustic modems (See Figure 1.) Two major field tests (FRONT2 and FRONT3) of the observation system were also completed and the final demonstration phase of the project (FRONT4) was been initiated. FRONT2 took place in the fall of 2000 and tested a subset of the 
observation array and telemetry network and whole array-network was deployed for the first time in the spring of 2001 during FRONT3. This deployment produced five four-month ADCP records. The CTD profilers were programmed to cycle every two hours and returned one 15 day and another 4 day record.
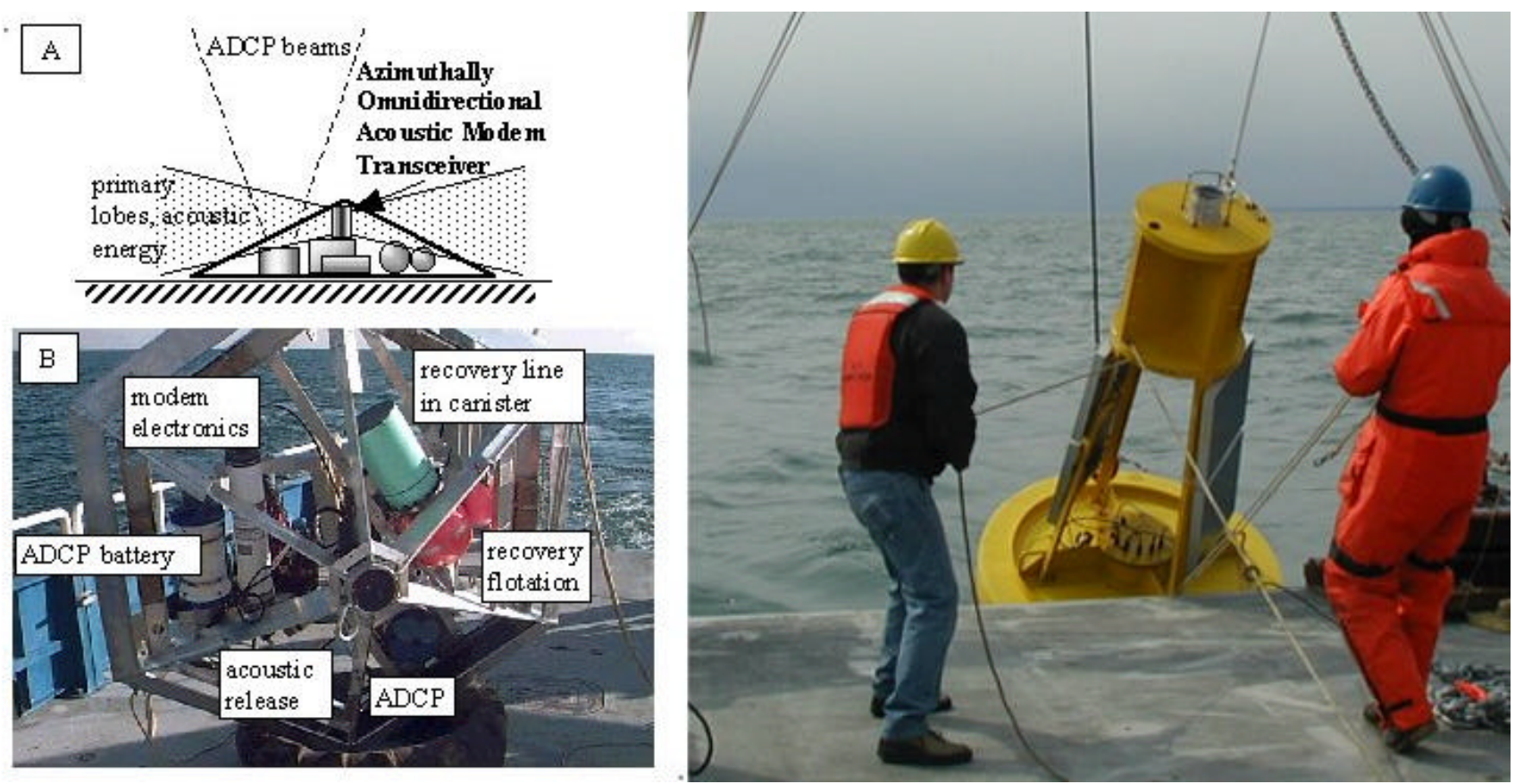

Figure 1. A schematic of the trawl-resistant "gazebo" ADCP-modem bottom frame (top left) with a photograph of it being deployed from the $R / V$ Connecticut below. The secondary gateway deployment is shown on the right.

The performance of Benthos's developments in modems and communications protocols were evaluated in FRONT3 in which a single ADCP-modem transmitted data to the gateway buoy every 40 minutes via five repeater modems at $\sim 1.2 \mathrm{~km}$ intervals. The value of the gateway's ability to monitor the performance of other modems by "eavesdropping" on their data transfer was demonstrated when two modems were lost to fishing activity. During this deployment calm winds and the short internode separation resulted in $85 \%$ if transmissions reaching shore successfully.

In FRONT3 we deployed 7 ADCP-modems, 7 repeater nodes, and a second Gateway buoy to augment USCG buoy. The internode separation was $3 \mathrm{~km}$ and the array spanned an area $18 \mathrm{~km} \times 12 \mathrm{~km}$. The modem protocol was improved to allow the receiving node to request retransmission of corrupt messages to increase the likelihood of success. In addition to demonstrating real time data delivery to shore with practical internode spacing, two-way communication between shore and an ADCP allowed its sampling parameters to be modified midway through the experiment thereby demonstrating the possibility of adaptive sampling.

During both FRONT3 and 4 large scale hydrographic and ADCP surveys were conducted by the R/V Connecticut to define the patterns of salinity, temperature and velocity in the mooring array and the surrounding waters. Two spring and two fall cruises spanned the range of conditions expected from an analysis of archived hydrographic data and river discharge records. Each of these survey cruises were followed by frontal survey using a short, towed array of five CTDs that deliver data to the deck at 0.3 $\mathrm{Hz}$ along a conducting cable to reveal $\sim 5 \mathrm{~m}$ structures. These observations guided the deployment of the turbulence sensor equipped REMUS ROV by Ed Levine. 
The modeling component of the project has advanced significantly in the last year. We have implemented the many improvements and new capabilities of the MITGCM in the FRONT application. The most important of these include: third order, upstream advection and nonlinear, flux-limited schemes yields better representation of small scale structures; implementation of the Large et al. (1994) vertical mixing parameterization produces a superior simulation of the vertical structure of tidal motion; and availability of an improved I/O facility allows for convenient comparison to available measurements.

We have invested considerable effort to construct initial and forcing fields for simulations. Tides are the primary source of energetic motion in the FRONT region and to improve the boundary forcing we have augmented the Egbert et al. (1997) predictions with observed sea level observations from New York and Atlantic City. Wind forcing is also significant and we have archived wind fields observed at Montauk Point Coast Guard station and compared them with those produced by the NCEP Eta-model with some general agreement. We have developed monthly climatologies of the vertical structure of temperature and salinity in the area to provide initial conditions. Although we employ thermal and fresh water forcing fields produced by the Eta-model, we are also examining observations for comparison. A weak nudging capability has been developed to smooth the interpolated forcing fields we derive from the spatially coarse, temporally sporadic AVHRR satellite observations.

The barotropic inverse model has now been implemented to provide subtidal boundary forcing estimates for the forward numerical model. We calculate the optimal solution to the depth-averaged, linear equations of motion constrained by bottom-moored ADCP records. This solution reveals a mean alongshore velocity field of $\sim 10 \mathrm{~cm} / \mathrm{s}$. Although this signal is relatively weak, it has substantial impact on the development of the frontal structures in the domain. Improvements to the barotropic inverse solution designed to account for nonlinear advection and more diverse data-types are currently being investigated.

Our approach to understanding and predicting the larger scale chlorophyll distribution and associated biological processes in the FRONT domain is, initially, to study a highly idealized ecosystem model with physical parameters from the regional general circulation modeling efforts. We are examining the quasisteady state scenarios for the summer to evaluate the extent to which the local physical-biological dynamics control the observed chlorophyll field. Recently published, related open ocean studies (Dutkiewicz et al., 2001; Follows and Dutkiewicz, 2001) provide tools and analysis procedures which will be adapted and applied.

To this end we have obtained physical fields from the regional circulation model runs and performed Lagrangian float simulations to examine the potential for local controls. These suggest that local dynamics can be influential and the chlorophyll distribution does not simply reflect advection from the surrounding region.

The in-situ biological profiling data along with a more detailed ecological analysis will provide a more detailed view of the vertical and temporal biological dynamics in frontal regions. We have developed simplified, two-dimensional frontal physical biological models with which to illustrate possible interactions of importance. These models incorporate a simplified, prescribed flowfield and a Lagrangian particle, individual based approach to ecosystem modeling (following A. Epstein, Ph.D. Thesis, MIT/WHOI Joint Program, 1995). These models will be applied to the FRONT region guided by the in situ data and understanding of the dynamics of the region from the physical and general circulation modeling studies. 


\section{RESULTS}

Substantial technological advances have been made in real-time data telemetry and modeling. According to the model, nonlinearly Island. This result is not surprising. However, Figure 2 shows the result of a passive tracer experiment rectified tidal flow occasionally reaches $20 \mathrm{~cm} / \mathrm{sec}$ in this domain. Velocities at tidal frequencies are much larger, and agree well with observed tides south of Block 10 days into the model run. The tracer reproduces many of the front-like features observed in satellite temperature and color. Although such features are generally associated with density variation and buoyancy effects, these results come from a homogeneous model run. In other words, the tidal residual flow appears to control the qualitative structure of the front-like features that motivated this study. This result is preliminary, and data analyses are underway to verify the dynamical mechanisms involved.

\section{IMPACT/APPLICATIONS}

Individual components of the FRONT project should be transportable to other regions. But the dynamical complexity of the FRONT site provides a special challenge to this kind of observation system. If we can develop predictive capabilities in this complex regime, then we should be able to translate the results to prediction of currents, the acoustic channel, and biology in the vicinity of coastal density fronts.

\section{TRANSITIONS}

The FRONT experiment schedule includes planned outages during which the fielded network may be used by SSC-SD to test prototype deployable sensors for surveillance, and other autonomous undersea devices, including mobile network nodes such as AUV's. The concomitant knowledge of the ocean structure provided by the FRONT system will allow SSC-SD to diagnose the relationships between network performance and the environment.

\section{RELATED PROJECTS}

FRONT is part of the National Oceanographic Partnership Program (NOPP). The project includes participation of scientists at University of Rhode Island (URI), National Undersea Warfare Center (NUWC) in Newport, Rhode Island, an SSC-SD. Dave Hebert (URI), Ed Levine (NUWC) and Joe Rice (SSC-SD) are the lead PI's on these related Awards.

\section{PUBLICATIONS}

Codiga, D. L., J. A. Rice, and P. S. Bogden, 2000. Real-time delivery of subsurface coastal circulation measurements from distributed instruments using networked acoustic modems. Proc. MTS/IEEE Oceans 2000, Vol 1, pp 575-582.

Dutkiewicz, S., Follows, M.J., J.C. Marshall and W.W. Gregg (2001) Interannual variability of phytoplankton abundance in the North Atlantic. Deep-Sea Res. II., 48, 2323-2344.

Follows, M.J., and S. Dutkiewicz (2001) Meteorological modulation of the North Atlantic spring bloom. In press, Deep-Sea Res. 